\title{
Estudio de la inversión de energía aplicada en la manufactura de instrumentos líticos recuperados en la costa rionegrina del golfo San Matías (Argentina) a lo largo del Holoceno medio y tardío
}

\author{
Jimena Alberti \\ Instituto Multidisciplinario de Historia y Ciencias Humanas (IMHICIHU) - Consejo Nacional de \\ Investigaciones Científicas y Técnicas (CONICET). Saavedra 15, 5to. piso. Buenos Aires, Argentina. \\ Email: jimealberti@gmail.com
}

\section{Resumen:}

La tecnología lítica es una de las estrategias que los grupos humanos del pasado pusieron en juego para, principalmente, extraer recursos del medio y procesarlos para el consumo. En este marco, diferentes artefactos pueden ser manufacturados invirtiendo distintas cantidades de energía (mientras que algunos requieren de un gran esfuerzo de producción, otros, en cambio, pueden ser producidos con muy poca inversión de energía), dependiendo de factores tales como la disponibilidad de materias primas, el fin para el cual se manufactura el instrumento, los constreñimientos temporales, entre otros. Esta inversión energética puede medirse a través de diferentes indicadores. Así, es esperable que en ambientes donde la variabilidad espacial y temporal da como resultado una distribución heterogénea de los recursos, los conjuntos de artefactos mostrarán mayor inversión de energía para ser más eficientes al momento de la explotación y procesamiento de recursos. El caso contrario se daría si la energía se ncuentra distribuida de forma homogénea en el ambiente (Torrence 1983; Jeske 1992; Bousman 1993).

El área de estudio de este trabajo comprende la costa del golfo San Matías (provincia de Río Negro, Argentina). Debido a las diferencias geomorfológicas y estructurales que la caracterizan, la costa rionegrina del golfo ha sido dividida en dos sectores distinguibles entre sí: el sector norte (entre San Antonio Oeste y el balneario El Cóndor), donde se combinan la accesibilidad a especies marinas, el agua dulce y la abundante disponibilidad de materias primas líticas y de reparos topográficos (Borella et al. 2007; Cardillo \& Favier Dubois 2010); y el sector oeste (entre Las Grutas y el límite con Chubut), ambientalmente más homogéneo y con una diversidad de especies marinas menor (Orensanz et al. 1973), utilizado de forma menos redundante o intensiva que la otra porción del golfo (Borella et al. 2007; Favier Dubois \& Borella 2011). Los fechados del sector norte se ubican entre los ca. 6000 y los 450 años 14C AP (Favier Dubois et al. 2009a), mientras que los del sector oeste entre los 3000 y los 900 años 14C AP (Borella et al. 2015).

Con el fin de distinguir esfuerzos diferenciales de manufactura, en este trabajo se presentan los resultados de la aplicación de los conceptos de "clase técnica” (Aschero \& Hocsman 2004) y "diseño utilitario" a los conjuntos líticos recuperados en el área de estudio. El primer concepto considera la superposición de los lascados que pueden cubrir total o parcialmente la superficie de una o de las dos caras, entrecruzándose o no en el eje medio del instrumento (Aschero \& Hocsman 2004). Las

Published by the School of History, Classics and Archaeology, University of Edinburgh ISSN: 2055-0472. URL: http://journals.ed.ac.uk/lithicstudies/

This work is licensed under a Creative Commons Attribution 2.5 UK: Scotland License. 
categorías determinadas dentro de la clase técnica no son compartimentos estancos sino que, de acuerdo con Hocsman \& Escola (2007: 76), existe un continuum en la morfología de los artefactos que va desde los que pueden ser producidos con muy poco esfuerzo tecnológico, hasta los que implican un mayor esfuerzo de producción. Esto se complementa con el estudio de los diseños utilitarios de los instrumentos. Mediante esta metodología se buscó distinguir esfuerzos diferenciales en la manufactura de las diversas clases artefactuales encontradas en los conjuntos analizados, y discernir tendencias respecto de la explotación de los diferentes tipos de rocas encontrados en el registro. Todos los análisis se realizaron siguiendo los criterios establecidos en Aschero (1975; 1983), mientras que para la identificación de las materias primas se estableció según Alberti \& Fernández (2015). Finalmente se hicieron análisis de Chi2 con el fin de determinar si las diferencias detectadas intra e intercosta en los diferentes bloques temporales resultaban ser estadísticamente significativas.

Para la costa norte del golfo, los resultados indican, en general, una baja inversión de energía en los momentos tempranos de la ocupación, acorde con la explotación de recursos marinos. Esto se apoya en los altos porcentajes de formas base para cuya obtención no habría sido necesario el uso de la percusión dirigida, en la mayoría de ausencia de requerimientos de formatización de la forma base, y en la ausencia de requerimientos de imposición de forma. Para momentos tardíos los resultados indican un aumento de esta inversión, en el marco de un aumento del riesgo y la diversificación de la dieta, ya que la manufactura de piezas mediante la técnica del adelgazamiento bifacial es mayor respecto a momentos tempranos. Esto se debería, posiblemente, a la aparición de nuevas tecnologías, como las puntas de proyectil, necesarias para la captura de alimentos terrestres.

Para la costa oeste del golfo, en cambio, la energía invertida en la manufactura de instrumentos se habría mantenido constante a lo largo del tiempo, predominando la baja inversión energética a lo largo de toda la ocupación.

Así, a través de la aplicación de los indicadores de inversión de energía, se pudo determinar que únicamente en la costa norte del golfo San Matías se habría dado un cambio en la inversión energética puesta en la manufactura de instrumentos a lo largo del tiempo, lo cual podría estar relacionado con la diversificación en la dieta propuesta para el área (Favier Dubois et al. 2009a). Sin embargo, dentro de esta baja inversión general, para momentos tempranos de la ocupación se habría dado una inversión energética ligeramente mayor en la costa oeste. Una explicación plausible para esto es la presentación de las materias primas y la calidad de las mismas para la talla: fuentes de rocas abundantes y ubicuas en el ambiente y de calidad regular a buena en la costa norte, y ubicadas en lugares puntuales del espacio y con rocas de mejor calidad en la costa oeste (Alberti 2015: 239-247). Así, podría pensarse que, debido a esfuerzos de aprovisionamiento más altos en la costa oeste, la tecnología tendería a ser de tipo conservada (sensu Nelson 1991), invirtiendo mayor energía en la manufactura de instrumentos con el fin de prolongar su vida útil. En la costa norte, en cambio, la amplia disponibilidad de rocas habría llevado al rápido descarte de los artefactos, en los que no se habría invertido gran energía.

Para momentos tardíos de la ocupación en el golfo, se registró un aumento en la inversión de energía únicamente en la costa norte, con lo que la incorporación de recursos terrestres a la dieta ocasionó variaciones solamente en esta región. Podría pensarse que para la costa oeste la energía invertida desde momentos tempranos y la tecnología conservada que se habría manejado en relación con el consumo de recursos y la presentación y disponibilidad de materias primas en el ambiente, habría sido suficiente para el aprovisionamiento, procesamiento y consumo de los nuevos recursos incorporados a la dieta en momentos tardíos.

A partir de este trabajo se pudo establecer un indicador más que reforzaría la idea de una ocupación más dinámica y cambiante en la costa norte, acorde con una distribución heterogénea de recursos alimenticios, y de un aprovechamiento más homogéneo del espacio en la costa oeste, concordante con una diversidad de recursos menor

Palabras clave: instrumentos líticos; clase técnica; diseño utilitario; golfo San Matías; Patagonia 


\section{Introducción}

La tecnología lítica es una de las estrategias que los grupos humanos pusieron en práctica en el pasado para resolver determinadas problemáticas y que hoy, gracias a la durabilidad de las rocas en el tiempo, puede ser abordada desde la arqueología. El estudio de la organización tecnológica de un grupo implica entender las estrategias que se pusieron en juego al momento de manufacturar, usar, transportar y descartar los instrumentos y los elementos que se necesitan para su producción y mantenimiento (Nelson 1991). Una forma de abordar la variabilidad interna de los conjuntos líticos es a través de la estimación de la inversión de trabajo puesta en la manufactura de las distintas clases artefactuales de los conjuntos arqueológicos (Bousman 1993). De acuerdo con Hocsman \& Escola (2007), algunos instrumentos requieren de un gran esfuerzo de producción, mientras que otros pueden ser producidos con muy poca inversión de energía. Es de esperar que en aquellos ambientes en donde la energía se distribuye homogéneamente, y pensando en términos de coste-beneficio, el trabajo invertido en la manufactura de los conjuntos líticos tendería a minimizarse, ya que no serían necesarios artefactos muy complejos para la explotación de los recursos disponibles en el ambiente. Por el contrario, en ambientes donde la variabilidad espacial y temporal distribuye los recursos de forma heterogénea, los conjuntos artefactuales mostrarán una mayor inversión de energía (por ejemplo, siendo más complejos y diversos) para incrementar su eficiencia en la explotación y procesamiento de recursos (Torrence 1983; Jeske 1992; Bousman 1993).

En este trabajo se presentan los resultados de la aplicación de la noción de "clase técnica” y del estudio de los diseños utilitarios en los conjuntos líticos recuperados en la costa rionegrina del golfo San Matías (Patagonia Argentina) poniendo el foco en la determinación de indicadores de inversión de trabajo diferencial entre materias primas distintas a lo largo del Holoceno medio y tardío.

\subsection{El área de estudio}

Debido a las diferencias geomorfológicas y estructurales que la caracterizan, la costa rionegrina del golfo ha sido dividida en dos sectores distinguibles entre sí. En el sector norte del golfo (entre San Antonio Oeste y el balneario El Cóndor) (Figura 1) se combinan la accesibilidad a especies marinas, el agua dulce y la abundante disponibilidad de materias primas líticas y de reparos topográficos (Borella et al. 2007; Cardillo \& Favier Dubois 2010). Los sitios localizados en este litoral fueron fechados entre los ca. 6000 y los 450 años ${ }^{14} \mathrm{C}$ AP (Favier Dubois et al. 2009a). Basado en evidencias artefactuales e isotópicas sobre restos esqueletales humanos, se ha propuesto para esta porción de la costa del golfo un modelo de consumo de recursos marinos que comprende tres etapas (Favier Dubois et al. 2009a): consumo intensivo (entre los $4800 \mathrm{y}$ los 2200 años ${ }^{14} \mathrm{C}$ AP, aunque en los sitios el consumo de mamíferos y aves marinas, crustáceos, moluscos y peces, se evidencia desde los 6000 años ${ }^{14}$ C AP (Favier Dubois et al. 2009a; Favier Dubois \& Scartascini 2012; Scartascini, 2010: 97100; 2014: 203-219); consumo moderado (entre 1500 y 450 años ${ }^{14} \mathrm{C} \mathrm{AP}$, con una disminución del componente marino en la dieta y un aumento del terrestre en el marco de un incremento del riesgo debido al aumento poblacional y la competencia por los recursos); y abandono, alrededor del siglo XVIII, en concordancia con la incorporación del caballo (Favier Dubois et al. 2009a). En esta porción del golfo las fuentes de materias primas son exclusivamente de tipo secundario, compuestas por rodados de diversas rocas (principalmente volcanitas intermedias-básicas y ácidas) de calidad regular a buena para la talla, y ubicuas en el ambiente (Alberti 2015: 92-96).

Por su parte, la costa oeste corre de norte a sur entre Las Grutas y hasta el límite con Chubut (Figura 1), y es ambientalmente más homogénea que la parte norte del golfo, con lo 
que presenta una diversidad de especies marinas menor (Orensanz et al. 1973). La menor disponibilidad de recursos, la baja presencia de agua dulce y la escasez de reparos topográficos, ha dado lugar a la propuesta de que esta zona habría sido usada de forma menos intensiva o no redundante, destacándose la presencia de ciertos enclaves atractivos para la ocupación humana (Borella et al. 2007; Favier Dubois \& Borella 2011). Esta hipótesis es congruente con lo observado en la diversidad y composición de los conjuntos líticos (Cardillo 2009: 133-135), y se refleja en la baja presencia de concheros, de evidencia bioarqueológica y faunística, y en la escasez de localidades utilizadas de forma repetida en el tiempo. Todo esto, sumado a la presencia de obsidiana proveniente de lugares distantes (Favier Dubois et al. 2009b), ha llevado a pensar que esta área podría haber funcionado en momentos tardíos como un espacio de circulación o de uso esporádico (o ambos), hipótesis que actualmente se está evaluando (Favier Dubois \& Borella 2011). Los fechados de los sitios de esta costa se ubican entre los 3000 y los 900 años ${ }^{14} \mathrm{C}$ AP (Borella et al. 2015) En esta costa hay tanto fuentes primarias como secundarias localizadas en lugares puntuales del espacio, de fácil acceso y explotación, con rocas de diversas calidades para la talla (Alberti 2015: 99-118), entre las que se cuentan las volcanitas ácidas, rocas piroclásticas, sedimentarias y variedades de sílex.

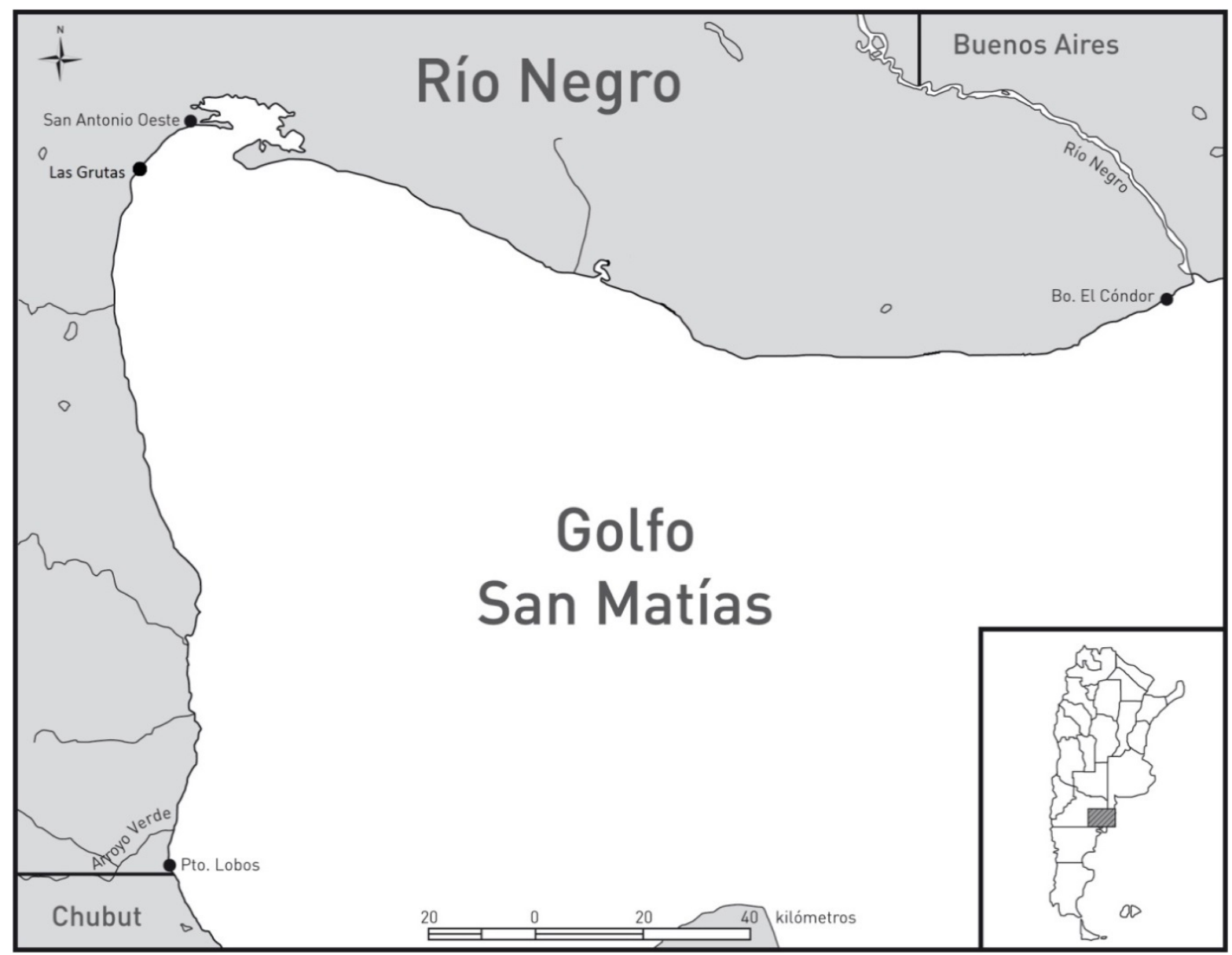

Figura 1. Costa rionegrina del golfo San Matías.

Figure 1. San Matías Gulf coast.

\section{Metodología}

Para estudiar la energía puesta en la manufactura de las diferentes clases artefactuales a partir de distintas rocas, en este trabajo se utilizan las nociones de "inversión de trabajo" (sensu Hocsman \& Escola 2007) y "clase técnica” (sensu Aschero \& Hocsman 2004). Mediante esta metodología se buscó distinguir esfuerzos diferenciales en la manufactura de 
las diversas clases artefactuales encontradas en los conjuntos analizados, y discernir tendencias respecto de la explotación de los diferentes tipos de rocas encontrados en el registro.

Para abordar con mayor exactitud la estimación de la inversión de trabajo en los artefactos líticos tallados, Aschero \& Hocsman (2004) han introducido la categoría analítica "clase técnica" que considera la superposición de los lascados que pueden cubrir total o parcialmente la superficie de una o de las dos caras, entrecruzándose o no en el eje medio del instrumento (Aschero \& Hocsman 2004). Es posible distinguir ocho clases técnicas en función de una inversión de trabajo creciente: artefacto con trabajo bipolar, artefacto con trabajo no invasivo alternante, artefacto con trabajo no invasivo unifacial, artefacto con trabajo no invasivo bifacial, artefacto con reducción unifacial, artefacto con adelgazamiento unifacial, artefacto con reducción bifacial, y artefacto con adelgazamiento bifacial (Aschero \& Hocsman 2004; Hocsman 2006: 76-77; Hocsman \& Escola 2007). Estas categorías no son compartimentos estancos sino que, de acuerdo con Hocsman \& Escola (2007: 76), existe un continuum en la morfología de los artefactos, que va desde los que pueden ser producidos con muy poco esfuerzo tecnológico, hasta los que implican un mayor esfuerzo de producción. Por otra parte, la clase técnica da cuenta únicamente de una parte de la inversión de trabajo puesta en un instrumento y sólo hace referencia a la formatización del mismo, con lo que es necesario tener en cuenta un mayor número de variables al momento de hacer el análisis. Así, para abordar el estudio de los diferentes diseños utilitarios, los autores proponen cuatro categorías analíticas (Hocsman \& Escola 2007):

- Clase técnica, tal como ya fue definida.

- Requerimientos de extracción de la forma base: se relaciona con la búsqueda, o no, de una forma base con caracteres específicos para su formatización posterior. Los autores diferencian entre percusión no dirigida y percusión dirigida (con mayor inversión de trabajo). En ambos casos hay intención de obtener una pieza adecuada; sin embargo, en el primero hay una amplia variedad de productos que pueden ser usados, mientras que en el segundo se busca obtener un producto concreto.

- Requerimientos de formatización de la forma base: implica la presencia de características de la forma base que inciden sobre la inversión de trabajo. Esta variable se especifica mediante presencia o ausencia.

- Requerimientos de imposición de forma: tiene que ver con la forma y dirección de los lascados de formatización o con la inversión de trabajo requerida para trabajar las diferentes partes de un instrumento. Esta variable también se especifica mediante presencia o ausencia.

Por otro lado, Hocsman \& Escola (2007) proponen un conjunto de variables que están en relación con las distintas categorías de clase técnica:

- Artefactos con adelgazamiento bifacial: piezas en las que la intencionalidad de los lascados, los cuales se extiendan desde el borde hasta el centro del artefacto y se superponen con los provenientes del margen opuesto, está puesta en rebajar el espesor y generar una sección regular biconvexa sin afectar el ancho del artefacto.

- Artefactos con reducción bifacial: confeccionados por lascados bifaciales que no cubren el centro de la cara o, de hacerlo, no se superponen con los que provienen del otro borde. El énfasis está puesto en la conformación de contornos.

- Artefactos con adelgazamiento unifacial: su confección se realiza por lascados que cubren sólo una de las caras. El objetivo es el mismo que en el adelgazamiento bifacial pero en una única cara del instrumento.

- Artefactos con reducción unifacial: la formatización unifacial afecta más a la cara que al borde y son respuesta a la búsqueda de contornos y secciones específicas. 
- Artefactos con trabajo no invasivo bifacial: el énfasis está en lograr un filo particular a partir de lascados en ambas caras, los cuales afectan más a los bordes. Tanto en esta categoría como en la siguiente, los lascados no alcanzan las zonas centrales.

- Artefactos con trabajo no invasivo unifacial: se busca una morfología de filo específica a partir de lascados que afectan el borde en una sola cara de las piezas.

- Artefactos con trabajo no invasivo alternante: en estos instrumentos se busca obtener un filo sin afectar las caras de la pieza, a través de la realización de lascados que se alternan en una y otra cara sobre un mismo borde.

- Artefactos con trabajo bipolar: en este caso hay escaso control sobre el producto final, con una baja inversión de energía en la manufactura. El objetivo principal es la búsqueda de filos.

Todos los análisis se realizaron siguiendo los criterios establecidos en Aschero (1975; 1983), mientras que para la identificación de las materias primas se realizó según Alberti \& Fernández (2015). Todas las medidas fueron tomadas con calibre digital y los datos volcados en planillas Excel confeccionadas para tal fin. Cabe destacar que por cuestiones de espacio se presentan en este trabajo únicamente los resultados del análisis de los instrumentos para las cuatro materias primas más utilizadas en la costa rionegrina (para mayor detalle ver Alberti 2015: 122-212). Finalmente se realizaron análisis de $\mathrm{Chi}^{2}$ con el fin de determinar si las diferencias detectadas intra e intercosta en los diferentes bloques temporales resultaban ser estadísticamente significativas. Para éstos se determinó un nivel de significación inferior a pvalor $<0.05$ (Hammer et al. 2001).

\section{Resultados}

\subsection{Costa norte}

\subsubsection{Bloque antiguo (6000-1500 años $A P)$}

Dentro de este bloque se realizaron 49 muestreos en 10 localidades arqueológicas de la costa norte. De los cuatro tipos de rocas mayormente utilizados, los instrumentos se dividieron de la siguiente manera (en la Tabla 1):

Tabla 1. Frecuencia y porcentaje de instrumentos distribuidos por materia prima para el bloque antiguo en la costa norte.

Table 1. Frequency and percentage of instruments divided according to raw materials for the northern coast earlier occupation span.

\begin{tabular}{lcc}
\hline & \multicolumn{2}{c}{ Instrumentos } \\
Materia Prima & $\mathbf{N}$ & $\mathbf{\%}$ \\
\hline Sílex & 44 & 35.77 \\
Volcanita intermedia- básica & 38 & 30.89 \\
Volcanita ácida & 21 & 17.07 \\
Calcedonia & 20 & 16.26 \\
\hline $\boldsymbol{\Sigma}$ & $\mathbf{1 2 3}$ & $\mathbf{1 0 0}$ \\
\hline
\end{tabular}

Como se desprende de la Tabla 1, el 36\% de la muestra corresponde a instrumentos de sílex, roca cuya presencia en las fuentes de materias primas del área es muy escasa (ca. 17\% sobre un total de 1.306 nódulos recuperados en los muestreos (Alberti 2015: 94). En segundo y tercer lugar siguen las rocas volcánicas en general, las cuales aparecen en las fuentes secundarias en porcentajes superiores al 60\%. En último lugar se ubican los instrumentos de calcedonia, roca alóctona en el área, cuyo porcentaje de instrumentos, sin embargo, es similar al de las volcanitas ácidas, de procedencia local. En relación con los tipos de filos, el 85\% de la muestra presenta filos simples, que es la categoría mayoritaria independientemente del tipo 
de roca que se considere. En orden de importancia siguen los filos de tipo bifacial (7\%), los dobles y finalmente los compuestos. Algunos de los artefactos se presentan en la Figura 2.

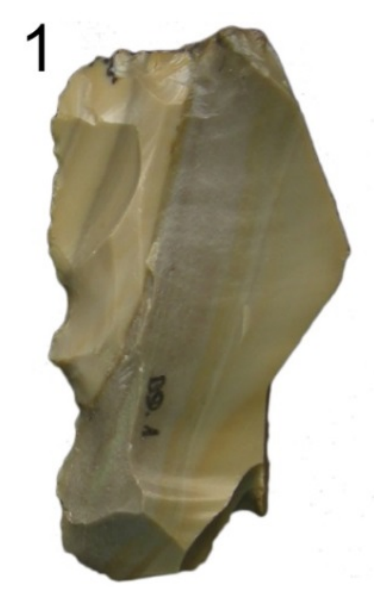

2
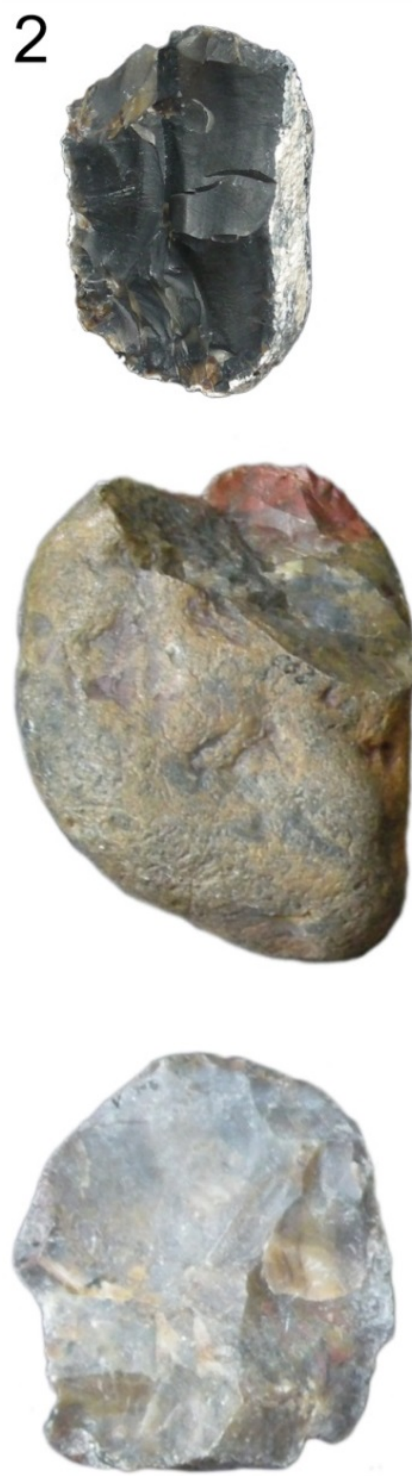
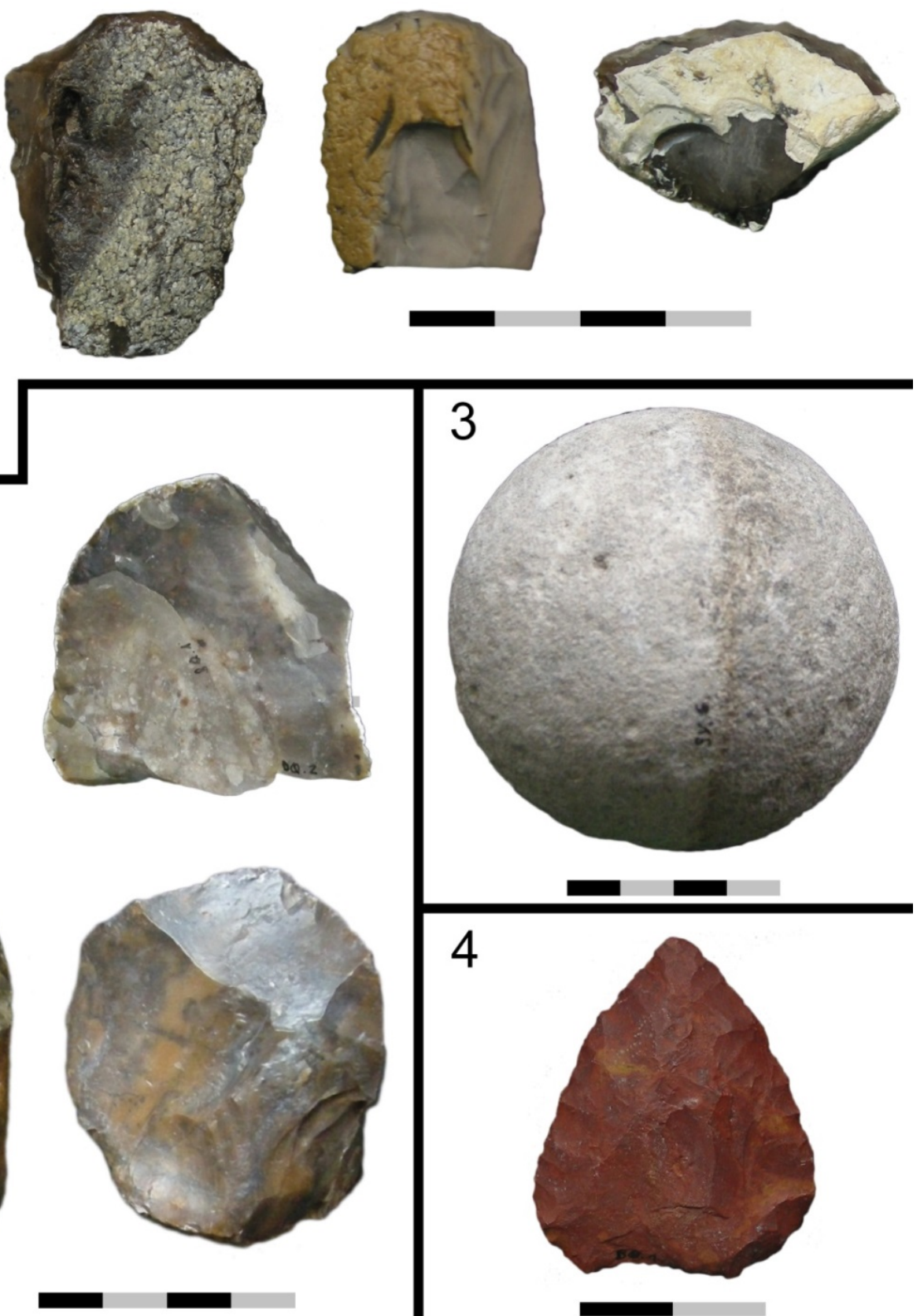

4
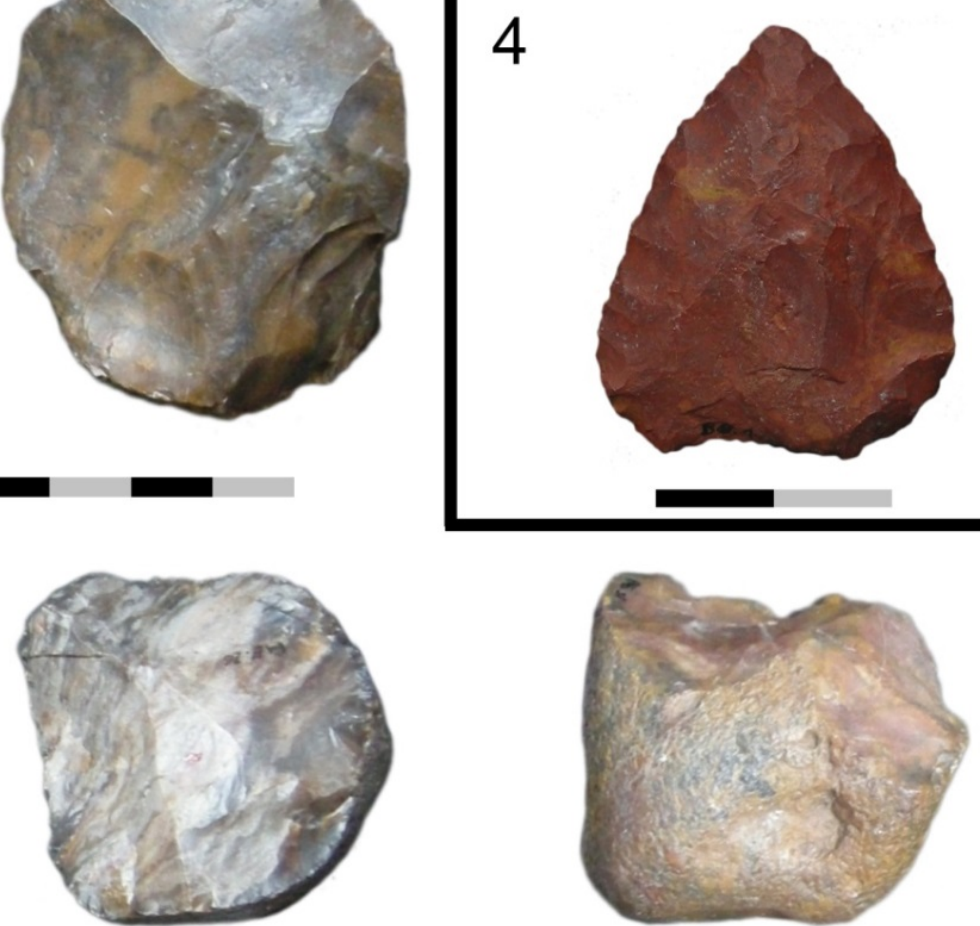

Figura 2. Artefactos recuperados en loci de la costa norte fechados en el bloque antiguo. 1. Raspadores y denticulados. 2. Núcleos. 3. Bola. 4. Punta de proyectil. Los segmentos de la escala representan $1 \mathrm{~cm}$.

Figure 2. Tools recovered at northern coast loci dated in the old span of marine resources consumption. 1. End scrapers and denticulates. 2. Cores. 3. Bola. 4. Projectile point. Scale bars are in $1 \mathrm{~cm}$ segments. 
Para determinar la energía invertida en la manufactura de los instrumentos analizados en el conjunto, se tomaron variables para definir la clase técnica y el diseño utilitario de los instrumentos. Los resultados de los análisis se presentan en la Tabla 2.

Tabla 2. Variables utilizadas para el estudio de la clase técnica y el diseño utilitario de los instrumentos de acuerdo con las materias primas determinadas en el conjunto. Volc. ác.: volcanitas ácidas. Volcanitas int-bas.: volcanitas intermedias-básicas. Req. ext.: requerimiento de extracción. PD: percusión dirigida. PND: percusión no dirigida. Req. form.: requerimiento de formatización. P: presente. A: ausente. Req. imp.: requerimiento de imposición. AB: adelgazamiento bifacial. RB: reducción bifacial. TNIB: trabajo no invasivo bifacial. TNIU: trabajo no invasivo unifacial. TB: trabajo bipolar.

Table 2. Variables used to study the technical class and utilitarian design of the instruments according to the raw materials. Volc. ác.: acidic volcanic rocks. Volcanitas int-bas.: intermediate-basic volcanic rocks. Req. ext.: extraction requirement. PD: direct percussion. PND: indirect percussion. Req. form.: manufacturing requirements. P: present. A: absent. Req. imp.: form imposition requirements. AB: bifacial thinning. RB: bifacial reduction. TNIB: non-invasive bifacial work. TNIU: non-invasive unifacial work. TB: bipolar work.

\begin{tabular}{lccccccccccc}
\hline $\begin{array}{l}\text { Materia } \\
\text { prima }\end{array}$ & \multicolumn{2}{c}{$\begin{array}{l}\text { Req. ext. de la } \\
\text { forma base }\end{array}$} & \multicolumn{2}{c}{$\begin{array}{c}\text { Req. form. de } \\
\text { la forma base }\end{array}$} & \multicolumn{2}{c}{$\begin{array}{c}\text { Req. imp. de } \\
\text { forma }\end{array}$} & \multicolumn{5}{c}{ Clase técnica } \\
& PD & PND & P & A & P & A & AB & RB & TNIB & TNIU & TB \\
\hline Sílex & 15 & 29 & 6 & 39 & 25 & 20 & 2 & 0 & 2 & 34 & 1 \\
Volcanitas & 6 & 27 & 3 & 31 & 11 & 22 & 1 & 1 & 1 & 27 & 1 \\
$\quad$ int.-bas. & & & & & & & & & & & \\
Volc. ác. & 2 & 2 & 9 & 3 & 9 & 3 & 0 & 1 & 2 & 12 & 0 \\
Calcedonia & 2 & 15 & 2 & 15 & 1 & 16 & 1 & 0 & 1 & 14 & 0 \\
\hline $\boldsymbol{\Sigma}$ & $\mathbf{2 5}$ & $\mathbf{7 3}$ & $\mathbf{2 0}$ & $\mathbf{8 8}$ & $\mathbf{4 6}$ & $\mathbf{6 1}$ & $\mathbf{4}$ & $\mathbf{2}$ & $\mathbf{6}$ & $\mathbf{8 7}$ & $\mathbf{2}$ \\
$\%$ & $\mathbf{2 5 . 5 1}$ & $\mathbf{7 4 . 4 9}$ & $\mathbf{1 8 . 5 1}$ & $\mathbf{8 1 . 4 9}$ & $\mathbf{4 2 . 5 9}$ & $\mathbf{5 7 . 4 1}$ & $\mathbf{3 . 9 6}$ & $\mathbf{1 . 9 8}$ & $\mathbf{5 . 9 4}$ & $\mathbf{8 6 . 1 3}$ & $\mathbf{1 . 9 8}$ \\
\hline
\end{tabular}

Según lo expresado en la Tabla 2, los instrumentos recuperados en estos conjuntos no habrían experimentado una alta inversión de energía en su manufactura. Esto se apoya por un lado en los porcentajes altos de formas base para cuya obtención no habría sido necesario el uso de la percusión dirigida (74\% del total); por otro lado, a la mayoría de ausencia de requerimientos de formatización de la forma base (81\% del total) y a la ausencia de requerimientos de imposición de forma ( $57 \%$ del conjunto). Finalmente, en lo referente a la clase técnica también se apoya esta noción ya que el $86 \%$ del conjunto de instrumentos fue manufacturado mediante trabajo no invasivo unifacial.

\subsubsection{Bloque reciente (1500-450 años $A P)$}

Dentro de este bloque se realizaron 31 muestreos en 9 localidades arqueológicas. De los cuatro tipos de rocas mayormente utilizados, los instrumentos se dividieron de la siguiente manera (en la Tabla 3):

Tabla 3. Frecuencia y porcentaje de instrumentos distribuidos por materia prima para el bloque reciente en la costa norte.

Table 3. Frequency and percentage of instruments divided according to raw materials for the northern coast later occupation span.

\begin{tabular}{lcc}
\hline & \multicolumn{2}{c}{ Instrumentos } \\
Materia Prima & $\mathbf{n}$ & $\mathbf{\%}$ \\
\hline Sílex & 45 & 55.55 \\
Volcanita intermedia-básica & 17 & 20.98 \\
Calcedonia & 15 & 18.51 \\
Volcanita ácida & 4 & 4.93 \\
\hline $\boldsymbol{\Sigma}$ & $\mathbf{8 1}$ & $\mathbf{1 0 0}$ \\
\hline
\end{tabular}



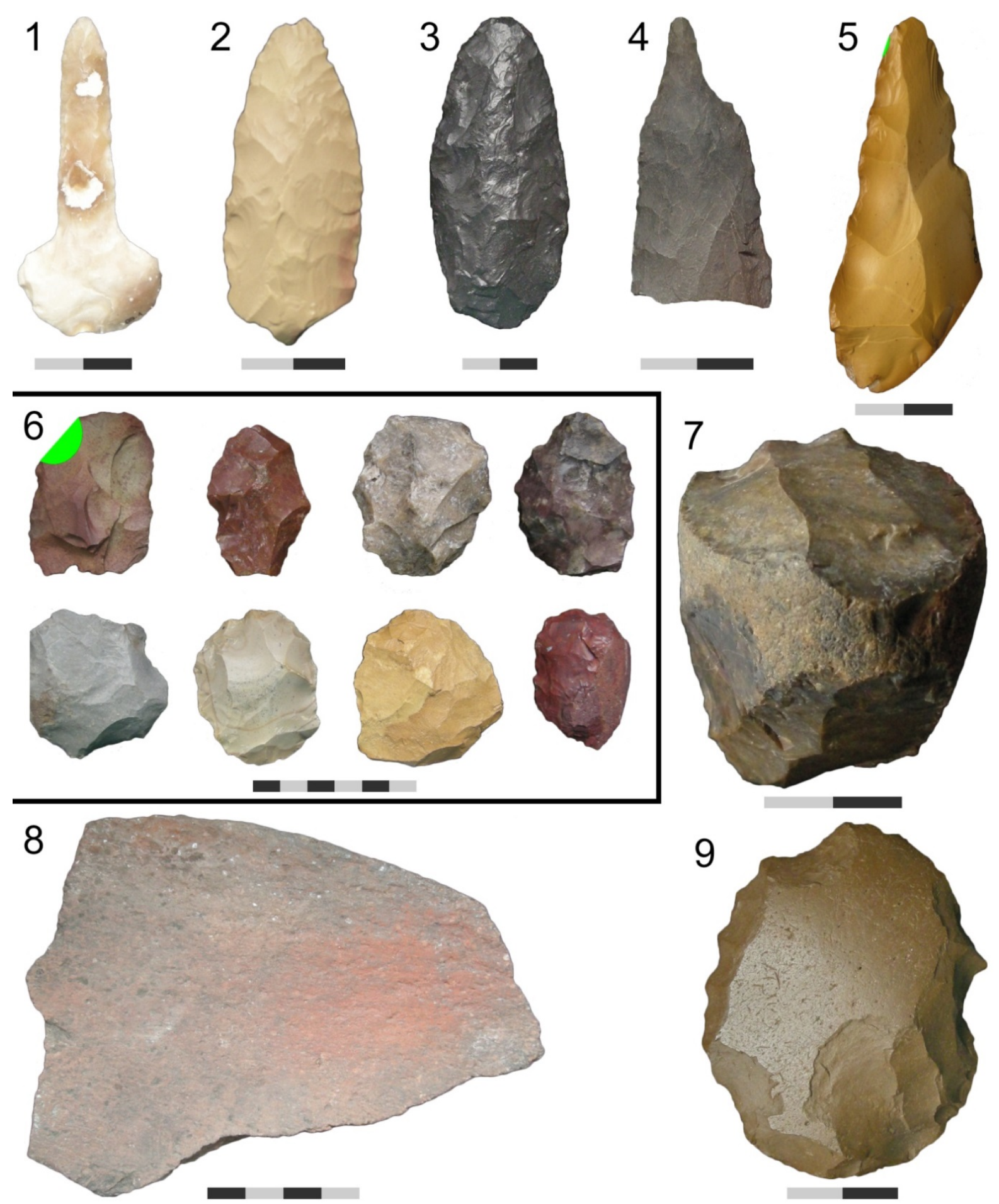

Figura 3. Artefactos recuperados en loci de la costa norte fechados en el bloque reciente. 1 y 4 . Perforadores. 2 y 3. Bifaces. 5. Denticulado. 6 y 7. Núcleos. 8. Molino con posibles restos de pigmento. 9. Denticulado sobre núcleo. (El color verde representa un fragmento de la imagen recortado debido a problemas técnicos). Los segmentos de la escala representan $1 \mathrm{~cm}$.

Figure 3. Tools recovered at northern coast loci dated in the recent block of marine resources consumption. 1 and 4. Perforators. 2 and 3. Bifaces tools. 5. Denticulate. 6 and 7. Cores. 8. Grinder with possible pigment remains. 9. Denticulate made using a core as base form. (Green colouring represents a part of the image cut off due to technical problems.) Scale bars are in $1 \mathrm{~cm}$ segments. 
Como se desprende de la Tabla 3, el 56\% de los instrumentos están manufacturados sobre sílex, lo cual es de destacar ya que a pesar de que esta muestra es más pequeña que la del bloque temprano, el número de instrumentos manufacturados sobre esta roca es mayor, lo cual podría indicar, junto con el aumento del uso de la calcedonia, una necesidad de utilizar rocas de mejor calidad para la talla debido a, posiblemente, el incremento del riesgo como consecuencia de un aumento poblacional, lo cual estaría relacionado, a su vez, con la diversificación en el consumo de recursos. Los instrumentos manufacturados sobre rocas volcánicas locales disminuyen sensiblemente su frecuencia para estos momentos. Respecto a la cantidad de filos, el $45 \%$ de la muestra presenta filos simples, que es la categoría mayoritaria independientemente del tipo de roca que se considere. En orden de importancia siguen los filos bifaciales (37\%), los dobles y los compuestos. Es de destacar que en este conjunto hay más tipos de filos que en los conjuntos previos, lo cual se debe, posiblemente, a la manufactura de mayor variedad de grupos tipológicos de artefactos. Algunos ejemplos de instrumentos recuperados se muestran en la Figura 3.

Los resultados de las variables tomadas para medir la inversión de energía en estos conjuntos se presentan en la Tabla 4. Las volcanitas ácidas no se contabilizaron ya que ninguno de los instrumentos analizados fue manufacturado mediante su talla.

Tabla 4. Variables utilizadas para el estudio de la clase técnica y el diseño utilitario de los instrumentos de acuerdo con las materias primas determinadas en el conjunto. Req. ext.: requerimiento de extracción. PD: percusión dirigida. PND: percusión no dirigida. Req. form.: requerimiento de formatización. P: presente. A: ausente. Req. imp.: requerimiento de imposición. AB: adelgazamiento bifacial. RB: reducción bifacial. RU: reducción unifacial. TNIB: trabajo no invasivo bifacial. TNIU: trabajo no invasivo unifacial.

Table 4. Variables used to study the technical class and utilitarian design of the instruments according to the raw materials. Req. ext.: extraction requirement. PD: direct percussion. PND: indirect percussion. Req. form.: manufacturing requirements. P: present. A: absent. Req. imp.: form imposition requirements. AB: bifacial thinning. RB: bifacial reduction. RU: unifacial reduction. TNIB: non-invasive bifacial work. TNIU: non-invasive unifacial work.

\begin{tabular}{|c|c|c|c|c|c|c|c|c|c|c|c|}
\hline \multirow[t]{2}{*}{$\begin{array}{l}\text { Materia } \\
\text { prima }\end{array}$} & \multicolumn{2}{|c|}{$\begin{array}{l}\text { Req. ext. de la } \\
\text { forma base }\end{array}$} & \multicolumn{2}{|c|}{$\begin{array}{l}\text { Req. form. de } \\
\text { la forma base }\end{array}$} & \multicolumn{2}{|c|}{$\begin{array}{l}\text { Req. imp. de } \\
\text { forma }\end{array}$} & \multicolumn{5}{|c|}{ Clase técnica } \\
\hline & PD & PND & $\mathbf{P}$ & A & $\mathbf{P}$ & A & $A B$ & RB & RU & TNIB & TNIU \\
\hline Sílex & 14 & 11 & 9 & 16 & 24 & 1 & 3 & 3 & 1 & 1 & 16 \\
\hline $\begin{array}{l}\text { Volcanitas } \\
\text { intermedia- } \\
\text { básica }\end{array}$ & 3 & 9 & 3 & 9 & 8 & 4 & 1 & 0 & 0 & 1 & 8 \\
\hline Calcedonia & 4 & 5 & 4 & 5 & 8 & 1 & 1 & 0 & 0 & 1 & 6 \\
\hline$\Sigma$ & 21 & 25 & 16 & 30 & 40 & 6 & 5 & 3 & 1 & 3 & 30 \\
\hline$\%$ & 45.65 & 54.35 & 34.78 & 65.22 & 86.95 & 13.04 & 11.9 & 7.14 & 2.38 & 7.14 & 71.42 \\
\hline
\end{tabular}

Según lo expresado en la Tabla 4, los instrumentos recuperados en estos conjuntos no habrían experimentado una alta inversión de energía en su manufactura, al igual que lo que se registró para el bloque anterior: 54\% del total corresponde a la ausencia de percusión dirigida para la obtención de las formas base, mientras que la falta de requerimientos de formatización de la forma base alcanza al 65\% de la muestra y la ausencia de requerimientos de imposición de forma al $87 \%$ del conjunto. Finalmente, en lo que se refiere a la clase técnica también se apoya esta noción ya que el $71 \%$ del conjunto de instrumentos fue manufacturado mediante trabajo no invasivo unifacial. Es de destacar, sin embargo, y a diferencia de lo que sucede en el bloque anterior, que el trabajo de las piezas mediante la técnica del adelgazamiento bifacial ocupa el segundo lugar en este conjunto, alcanzando un $12 \%$ de la muestra total. Esto se debería, posiblemente, a la aparición de nuevas tecnologías, como las puntas de proyectil, necesarias para la captura de animales terrestres. 


\subsubsection{Breve comparación temporal}

Para determinar si las diferencias en los esfuerzos de manufactura de los instrumentos detectadas entre ambos momentos de la ocupación de la costa norte son estadísticamente significativas, se realizaron análisis de $\mathrm{Chi}^{2}$ tomando las variables usadas para definir los diseños utilitarios. Como ya se aclaró previamente, el nivel de significación tomado fue de pvalor $<0.05$. Para la costa norte, todos los análisis dieron significativos. En los casos de los requerimientos de extracción y de formatización de la forma base, los valores de $\mathrm{Chi}^{2}$ fueron de 5.84 y 4.76 respectivamente, lo cual indicaría que a pesar de la diferencia en el tamaño de la muestra, la frecuencia de instrumentos manufacturados para los cuales no se habría requerido una forma base particular, ni que debiera ser formatizada previamente, es diferente entre los dos bloques (el tamaño de muestra no estaría influenciando estos valores). En el caso de los requerimientos de imposición de forma, los resultados están invertidos entre ambos bloques, es decir, en el bloque temprano hay mayor ausencia, mientras que en el reciente hay mayor presencia, lo que indicaría un esfuerzo de manufactura mayor. El análisis dio como resultado $\mathrm{Chi}^{2}=25.74$, lo cual indicaría que las diferencias entre ambos bloques temporales no se deben al azar y podrían responder a una mayor inversión de energía en la manufactura.

\subsection{Costa oeste}

\subsubsection{Bloque antiguo (3000-1500 años AP)}

Dentro de este bloque se realizaron 25 muestreos en siete localidades arqueológicas de la costa oeste. De los cuatro tipos de rocas mayormente utilizados, los instrumentos se dividieron de la siguiente manera (en la Tabla 5):

Tabla 5. Frecuencia y porcentaje de instrumentos distribuidos por materia prima para el bloque antiguo en la costa oeste.

Table 5. Frequency and percentage of instruments divided according to raw materials for the western coast earlier occupation span.

\begin{tabular}{lcc}
\hline & \multicolumn{2}{c}{ Instrumentos } \\
Materia prima & $\mathbf{n}$ & $\mathbf{\%}$ \\
\hline Calcedonia & 23 & 35.38 \\
Sílex & 20 & 30.77 \\
Volcanita ácida & 19 & 29.23 \\
Volcanita intermedia- básica & 3 & 4.62 \\
\hline $\boldsymbol{\Sigma}$ & $\mathbf{6 5}$ & $\mathbf{1 0 0}$ \\
\hline
\end{tabular}

Como se desprende de la Tabla 5, la roca más utilizada para la manufactura de instrumentos en el bloque antiguo de la costa oeste es la calcedonia (35\%), seguida del sílex (31\%) y las rocas volcánicas en tercer y cuarto lugar. Cabe destacar que en los muestreos de materias primas del área, sobre un total de 339 nódulos recuperados, el $45 \%$ corresponde a sílex, el $17 \%$ a las rocas volcánicas ácidas, el 9\% a calcedonia y menos del 1\% a volcanitas intermedias-básicas, por lo que se podría pensar en un uso local de materias primas, al margen de su calidad para la talla. Respecto de los filos, el 61\% de la muestra presenta filos simples y el 25\% filos bifaciales. Los instrumentos con filos dobles y compuestos se ubican en tercer y cuarto lugar. Algunos artefactos de estos conjuntos se muestran en la Figura 4. 

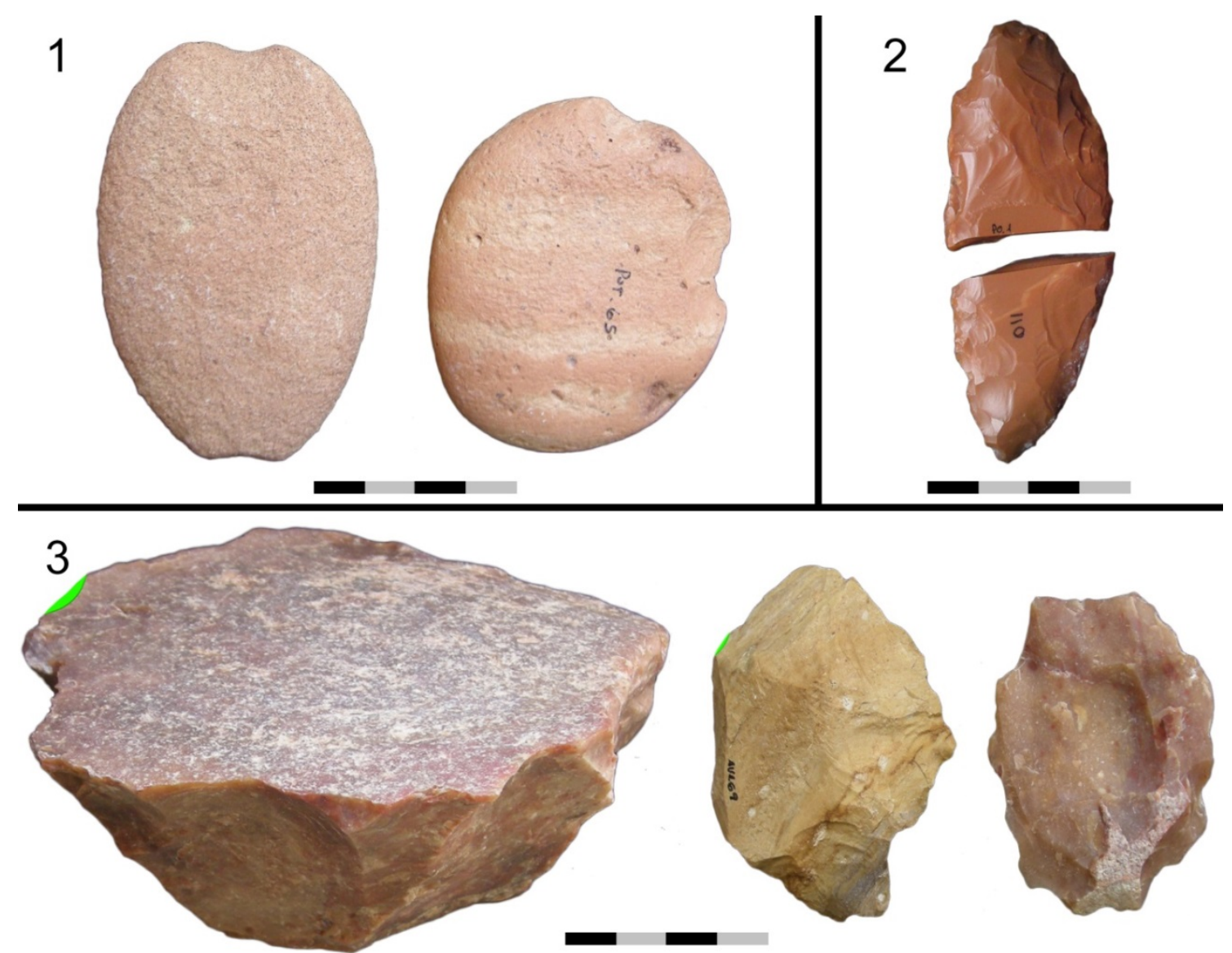

Figura 4. Artefactos recuperados en loci fechados en el bloque antiguo de la costa oeste. 1. Pesas líticas. 2. Bifaz. 3. Núcleos. (El color verde representa un fragmento de la imagen recortado debido a problemas técnicos). Los segmentos de la escala representan $1 \mathrm{~cm}$.

Figure 4. Tools recovered at western coast loci dated in the old block of marine resources consumption. 1. Weights. 2. Biface tool. 3. Cores. (Green colouring represents parts of the image cut off due to technical problems.) Scale bars are in $1 \mathrm{~cm}$ segments.

Las variables analizadas se distribuyeron de la siguiente manera en la Tabla 6:

Tabla 6. Variables utilizadas para el estudio de la clase técnica y el diseño utilitario de los instrumentos de acuerdo con las materias primas determinadas en el conjunto. Volc.: volcanitas. Req. ext.: requerimiento de extracción. PD: percusión dirigida. PND: percusión no dirigida. Req. form.: requerimiento de formatización. P: presente. A: ausente. Req. imp.: requerimiento de imposición. AB: adelgazamiento bifacial. RB: reducción bifacial. AU: adelgazamiento unifacial. TNIB: trabajo no invasivo bifacial. TNIU: trabajo no invasivo unifacial. Table 6. Variables used to study the technical class and utilitarian design of the instruments according to the raw materials. Volc.: volcanic rocks. Req. ext.: extraction requirement. PD: direct percussion. PND: indirect percussion. Req. form.: manufacturing requirements. P: present. A: absent. Req. imp.: form imposition requirements. AB: bifacial thinning. RB: bifacial reduction. AU: unifacial thinning. TNIB: non-invasive bifacial work. TNIB: non-invasive bifacial work. TNIU: non-invasive unifacial work.

\begin{tabular}{lccccccccccc}
\hline $\begin{array}{l}\text { Materia } \\
\text { prima }\end{array}$ & \multicolumn{2}{c}{$\begin{array}{c}\text { Req. Ext. De } \\
\text { la forma } \\
\text { base }\end{array}$} & \multicolumn{1}{c}{$\begin{array}{c}\text { Req. Form.de } \\
\text { la forma base }\end{array}$} & \multicolumn{2}{c}{$\begin{array}{c}\text { Req. Imp. } \\
\text { De forma }\end{array}$} & \multicolumn{4}{c}{ Clase técnica } \\
& PD & PND & P & A & P & A & AB & RB & AU & TNIB & TNIU \\
\hline Calcedonia & 12 & 12 & 6 & 18 & 18 & 6 & 1 & 3 & 0 & 2 & 12 \\
Sílex & 12 & 8 & 9 & 11 & 18 & 2 & 4 & 4 & 0 & 1 & 9 \\
Volc. ácida & 4 & 2 & 1 & 7 & 5 & 5 & 0 & 0 & 1 & 0 & 8 \\
\hline $\boldsymbol{\Sigma}$ & $\mathbf{2 8}$ & $\mathbf{2 2}$ & $\mathbf{1 6}$ & $\mathbf{3 6}$ & $\mathbf{4 1}$ & $\mathbf{1 3}$ & $\mathbf{5}$ & $\mathbf{7}$ & $\mathbf{1}$ & $\mathbf{3}$ & $\mathbf{2 9}$ \\
$\%$ & $\mathbf{5 6}$ & $\mathbf{4 4}$ & $\mathbf{3 0 . 7 6}$ & $\mathbf{6 9 . 2 3}$ & $\mathbf{7 5 . 9 2}$ & $\mathbf{2 4 . 0 8}$ & $\mathbf{1 1 . 1 1}$ & $\mathbf{1 5 . 5 5}$ & $\mathbf{2 . 2 2}$ & $\mathbf{6 . 6 6}$ & $\mathbf{6 4 . 4 4}$ \\
\hline
\end{tabular}


Según lo expresado en la Tabla 6, los instrumentos recuperados en estos conjuntos habrían experimentado una inversión de energía mayor en su manufactura que los instrumentos recuperados en el mismo bloque para la costa norte. Esto responde por un lado a los altos porcentajes de formas base para cuya obtención habría sido necesario el uso de la percusión dirigida (56\%); por otro lado, a la mayoría de la necesidad de requerimientos de imposición de forma (69\%). En lo que hace referencia a la clase técnica, en cambio, los datos recuperados abogan en favor de instrumentos manufacturados mediante una baja inversión de energía, ya que la que predomina es el trabajo no invasivo unifacial (64\%).

\subsubsection{Bloque reciente (1500-900 años $A P)$}

Dentro de este bloque se realizaron 25 muestreos en 11 localidades arqueológicas de la costa oeste.

En estos conjuntos, el orden de importancia de las materias primas utilizadas es el mismo que en el caso del bloque temprano de la costa oeste: predominan los instrumentos de calcedonia y sílex (35 y 31\%, respectivamente), seguidos de los de rocas volcánicas en general (ver Tabla 7). Cabe destacar que, a pesar de que la muestra es menor que la de momentos tempranos, el aumento en el número de instrumentos de calcedonia es importante. Respecto de los filos, el $69 \%$ de la muestra presenta filos simples, que es la categoría mayoritaria independientemente del tipo de roca que se considere. En orden de importancia siguen los instrumentos con filos de tipo bifacial (12\%), los dobles y finalmente los compuestos. Algunos ejemplos de estos instrumentos se muestran en la Figura 5.

Tabla 7. Frecuencia y porcentaje de artefactos distribuidos por materia prima para el bloque reciente en la costa oeste.

Table 7. Frequency and percentage of instruments divided according to raw materials for the western coast later occupation span.

\begin{tabular}{lcc}
\hline & \multicolumn{2}{c}{ Instrumentos } \\
Materia prima & $\mathbf{n}$ & $\mathbf{\%}$ \\
\hline Calcedonia & 31 & 56.36 \\
Sílex & 18 & 32.73 \\
Volcanita ácida & 5 & 9.09 \\
Volcanita intermedia- básica & 1 & 1.82 \\
\hline $\boldsymbol{\Sigma}$ & $\mathbf{5 5}$ & $\mathbf{1 0 0}$ \\
\hline
\end{tabular}



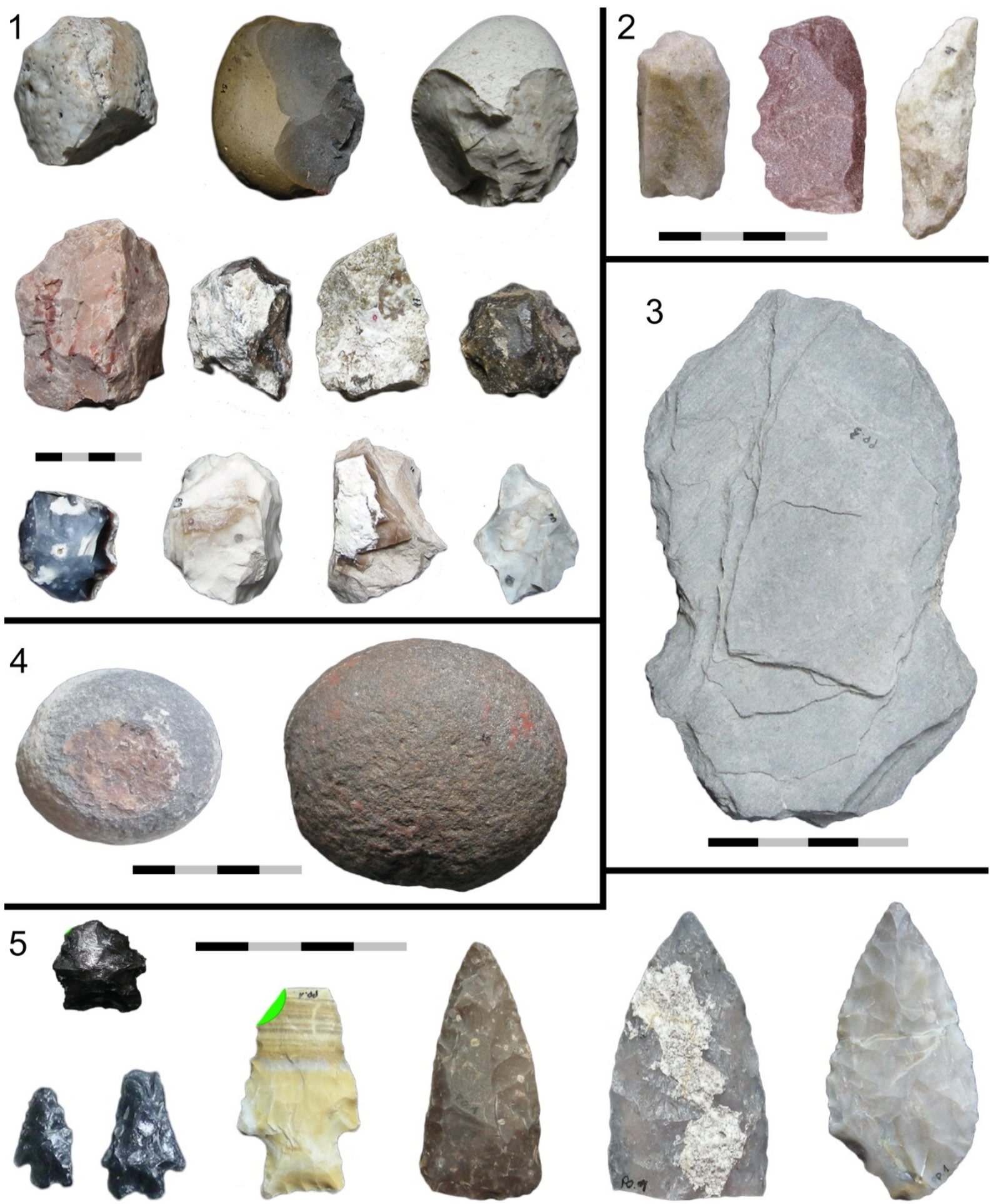

Figura 5. Artefactos recuperados en loci fechados en el bloque reciente de la costa oeste. 1. Núcleos. 2. Raspador, denticulado y cuchillo. 3. Posible hacha. 4. Bolas.5. Puntas de proyectil. (El color verde representa un fragmento de la imagen recortado debido a problemas técnicos). Los segmentos de la escala representan $1 \mathrm{~cm}$.

Figure 5. Tools recovered at western coast loci dated in the recent block of marine resources consumption. 1 . Cores. 2. Denticulate, end-scraper and knife. 3. possible axe. 4. Bolas. 5. Projectile points. (Green colouring represents a part of the image cut off due to technical problems.) Scale bars are in $1 \mathrm{~cm}$ segments. 
Las variables para determinar la inversión de energía puesta en la manufactura de estos instrumentos arrojaron los siguientes resultados en la Table 8:

Tabla 8. Variables utilizadas para el estudio de la clase técnica y el diseño utilitario de los instrumentos de acuerdo con las materias primas determinadas en el conjunto. Volc.: volcanita. Int: intermedia. Req. ext.: requerimiento de extracción. PD: percusión dirigida. PND: percusión no dirigida. Req. form.: requerimiento de formatización. P: presente. A: ausente. Req. imp.: requerimiento de imposición. AB: adelgazamiento bifacial. RB: reducción bifacial. RU: reducción unifacial. TNIB: trabajo no invasivo bifacial. TNIU: trabajo no invasivo unifacial.

Table 8. Variables used to study the technical class and utilitarian design of the instruments according to the raw materials. Volc.: volcanic rocks. Int.: intermediate rock. Req. ext.: extraction requirement. PD: direct percussion. PND: indirect percussion. Req. form.: manufacturing requirements. P: present. A: absent. Req. imp.: form imposition requirements. AB: bifacial thinning. RB: bifacial reduction. RU: unifacial reduction. TNIB: noninvasive bifacial work. TNIU: non-invasive unifacial work.

\begin{tabular}{|c|c|c|c|c|c|c|c|c|c|c|c|}
\hline \multirow[t]{2}{*}{$\begin{array}{l}\text { Materia } \\
\text { prima }\end{array}$} & \multicolumn{2}{|c|}{$\begin{array}{l}\text { Req. ext. de } \\
\text { la forma base }\end{array}$} & \multicolumn{2}{|c|}{$\begin{array}{c}\text { Req. } \\
\text { form. de } \\
\text { la forma } \\
\text { base }\end{array}$} & \multicolumn{2}{|c|}{$\begin{array}{l}\text { Req. imp. de } \\
\text { forma }\end{array}$} & \multicolumn{5}{|c|}{ Clase técnica } \\
\hline & PD & PND & $\mathbf{P}$ & A & $\mathbf{P}$ & A & $A B$ & RB & RU & TNIB & TNIU \\
\hline Calcedonia & 15 & 15 & 6 & 23 & 25 & 5 & 1 & 7 & 1 & 0 & 18 \\
\hline Sílex & 7 & 11 & 2 & 16 & 14 & 4 & 0 & 0 & 1 & 3 & 12 \\
\hline Volc. ácida & 0 & 2 & 1 & 1 & 2 & 0 & 0 & 0 & 0 & 0 & 2 \\
\hline $\begin{array}{l}\text { Volcanita } \\
\text { int-básica }\end{array}$ & 0 & 1 & 0 & 1 & 0 & 1 & 0 & 0 & 0 & 0 & 1 \\
\hline$\Sigma$ & 22 & 29 & 9 & 41 & 41 & 10 & 1 & 7 & 2 & 3 & 33 \\
\hline$\%$ & 43.14 & 56.86 & 18 & 82 & 80.39 & 19.61 & 2.17 & 15.22 & 4.35 & 6.52 & 71.74 \\
\hline
\end{tabular}

Según lo expresado en la Tabla 8, la única variable indicativa de una alta inversión de energía se observa en la presencia mayor de instrumentos con requerimiento de imposición de forma (80\%). El resto de las variables consignadas, dejan ver instrumentos en los que no se invirtió una alta energía para manufacturarlos, ya que tanto en los requerimientos de extracción, como en los de formatización de la forma base, los valores más altos son los que indican una baja inversión de energía. En lo que se refiere a la clase técnica, también se aprecia esta tendencia, ya que la clase técnica que predomina en los conjuntos es el trabajo no invasivo unifacial (72\%).

\subsubsection{Breve comparación temporal}

A partir de la realización de los análisis estadísticos, se pudo determinar que ninguna de las diferencias detectadas en las diferentes variables utilizadas para el estudio de los diseños utilitarios es estadísticamente significativa. Es decir, que a pesar de que hay diferencias en las frecuencias de instrumentos en las distintas categorías, éstas no indicarían una mayor o menor inversión de energía sino que ésto se debería a cuestiones relacionadas con el azar.

\subsection{Comparación inter-costa entre bloques temporales}

De acuerdo con lo expuesto, entonces, las diferencias estadísticamente significativas en relación con la inversión de energía puesta para manufacturar instrumentos en la costa rionegrina a lo largo del tiempo se dan únicamente para la porción norte del golfo, mientras que en la porción oeste las tendencias se habrían mantenido constantes durante la ocupación del área. Con el fin de determinar si las tendencias fueron distintas entre costas para el mismo rango temporal, también se realizaron análisis de $\mathrm{Chi}^{2}$. En el caso del bloque antiguo, las 
diferencias estadísticamente significativas se detectaron para el caso de los requerimientos de extracción de la forma base $\left(\mathrm{Chi}^{2}=13.39\right)$ y para el de los de imposición de forma $\left(\mathrm{Chi}^{2}=16.08\right)$, mientras que las diferencias en los requerimientos de formatización de la forma base se deberían a cuestiones relacionadas con el azar.

Para momentos tardíos de la ocupación, ningún indicador arrojó resultados estadísticamente significativos, con lo que podría afirmarse que las diferencias en las frecuencias de instrumentos detectadas entre costas puede deberse a factores relacionados con el azar. En ambas costas, entonces, las tendencias en la inversión de energía para la manufactura de instrumentos en momentos tardíos de la ocupación serían similares. El único indicador de una inversión de energía mayor para este período en la costa rionegrina sería la presencia mayoritaria de requerimientos de imposición de forma en la talla de los instrumentos de piedra.

\section{Discusión y conclusiones}

A través de la aplicación de los indicadores de inversión de energía se pueden dilucidar tendencias en la manufactura de instrumentos líticos a lo largo de la ocupación de la costa rionegrina. Durante el bloque antiguo en la costa norte, en el cual se ha planteado que la tecnología habría sido de tipo expeditiva en concordancia con la explotación de recursos marinos que habrían requerido de instrumentos simples para su aprovechamiento (Favier Dubois et al. 2009a), predominan los filos simples en todos los tipos de materias primas analizados. Es decir, que más allá de su procedencia, estas rocas habrían sido aprovechadas de forma similar, sin distinguir esfuerzos de manufactura diferenciales. En orden de importancia siguen los filos bifaciales, los cuales se registraron tanto en rocas locales (volcanitas) como en rocas alóctonas para el área, lo cual indicaría que, a pesar de que la proporción de filos bifaciales es más importante que la de por ejemplo, los dobles, ésto no estuvo relacionado con una explotación más intensa de rocas no locales. Los indicadores de la inversión de trabajo y la clase técnica también apoyan la idea de una baja intensidad de aprovechamiento de las materias primas, ya que predominan la percusión no dirigida para la extracción de las formas base, la ausencia de requerimiento de formatización de la forma base y de imposición de forma, y el trabajo no invasivo unifacial en todas las materias primas, independientemente de su calidad para la talla.

Para el bloque reciente de la misma costa, y en el marco de una diversificación en el consumo de recursos que habría requerido de tecnologías más complejas y con mayor inversión energética para su explotación, la diferencia registrada entre el porcentaje de filos simples y bifaciales se ve sensiblemente reducida, indicando un aumento de este segundo tipo de filos. En relación con los indicadores analizados, para este caso se registró también una baja inversión de energía, ya que predomina la percusión no dirigida para la extracción de las formas base, hay ausencia de formatización de éstas y la clase técnica principal es el trabajo no invasivo unifacial. Sin embargo, sí hay mayor porcentaje de presencia de requerimientos de imposición de forma, lo que podría estar relacionado con la aparición de mayor cantidad de puntas de proyectil y artefactos bifaciales en general. En el caso de los requerimientos de extracción de la forma base y de la formatización de la misma, para el bloque tardío se registró una disminución en el porcentaje de indicadores que señalan baja inversión de energía (percusión no dirigida y ausencia de formatización de la forma base) y un aumento en los indicadores que señalan mayor inversión energética. A su vez, la presencia de requerimientos de imposición de forma es mayor en el bloque tardío y disminuye la cantidad de artefactos formatizados mediante trabajo no invasivo unifacial. Así, se puede observar que, a pesar de que en general sigue predominando para momentos tardíos una baja inversión de energía, hay indicadores que señalarían un aumento en el esfuerzo de manufactura de los instrumentos. 
Los análisis estadísticos realizados para esta costa dieron resultados significativos, lo cual implica que estas variaciones en los porcentajes no responden al azar y que, en cambio, podrían estar relacionadas con factores que podrían responder a un cambio en la manufactura de artefactos debido a la necesidad de contar con nuevas tecnologías para aprovechar los recursos que se comenzaron a incorporar a la dieta.

Para la costa oeste, donde las fuentes de materias primas líticas se presentan de forma diferente, en el bloque temprano predominan los instrumentos con filos simples para todas las materias primas, posicionándose los filos bifaciales en segundo lugar. En relación con los indicadores registrados en este trabajo, en el bloque temprano, y a diferencia de lo que sucede en la otra región, en la costa oeste predomina la percusión dirigida para la extracción de las formas base y hay mayor presencia de requerimientos de imposición de forma, aunque prevalece la ausencia de requerimientos de formatización de la forma base. La clase técnica tampoco sustenta la idea de una mayor inversión energética, ya que la principal es el trabajo no invasivo unifacial.

Para el bloque reciente de la costa oeste los filos simples son mayoría también, seguido de los bifaciales, pero éstos en un porcentaje mucho menor que para momentos tempranos. Respecto de los indicadores propuestos, predomina la percusión no dirigida, al contrario que para momentos tempranos, la ausencia de formatización de la forma base y el trabajo no invasivo unifacial. El único indicador que apuntaría a una inversión energética mayor, al igual que para momentos previos, es el de los requerimientos de imposición de forma, cuyo porcentaje es el mayor del conjunto. Los análisis estadísticos dieron como resultado que las diferencias detectadas entre ambos bloques temporales en esta costa no son significativas, por lo que podrían deberse a cuestiones relacionadas con el azar, tal como por ejemplo unas intensidades de muestreo diferentes.

De este modo, se pudo determinar que únicamente en la costa norte del golfo San Matías se habría dado un cambio en la inversión energética puesta en la manufactura de instrumentos a lo largo del tiempo, lo cual podría estar relacionado con la diversificación en la dieta propuesta para el área (Favier Dubois et al. 2009a). Para momentos tempranos de la ocupación de la costa rionegrina, se habría dado una inversión energética mayor en la costa oeste, donde la proporción de presencia de requerimientos de formatización de la forma base y de imposición de forma es mayor. En toda la región habría existido una dieta predominantemente marina, con lo que es necesario buscar la explicación de una mayor inversión de energía en otros factores. Una explicación plausible es la presentación de las materias primas y la calidad de las mismas para la talla: mientras que en la costa norte las fuentes son abundantes y ubicuas en el ambiente y de calidad regular a buena para la talla, en la costa oeste se ubican en lugares puntuales en el espacio y presentan rocas de mejor calidad (Alberti 2015: 239-247). Así, podría pensarse que, debido a esfuerzos de aprovisionamiento más altos en la costa oeste, la tecnología tendería a ser de tipo conservada (sensu Nelson 1991), invirtiendo mayor energía en la manufactura de instrumentos con el fin de prolongar su vida útil. En la costa norte, en cambio, la amplia disponibilidad de rocas habría llevado a los grupos a descartar rápidamente los artefactos, en los que no se habría invertido gran energía.

Para momentos tardíos de la ocupación en el golfo, se registró un aumento en la inversión de energía únicamente en la costa norte, con lo que la incorporación de recursos terrestres a la dieta ocasionó variaciones solamente en esta costa. De esta forma, podría pensarse que para el caso de la costa oeste la energía invertida desde momentos tempranos y la tecnología conservada que se habría manejado en relación con el consumo de recursos y la presentación y disponibilidad de materias primas en el ambiente, habría sido suficiente para el aprovisionamiento, procesamiento y consumo de los nuevos recursos incorporados a la dieta en momentos tardíos. 
A partir de este trabajo se pudo establecer un indicador más que reforzaría la idea de una ocupación más dinámica y cambiante en la costa norte, acorde con una distribución heterogénea de recursos alimenticios, y de un aprovechamiento más homogéneo del espacio en la costa oeste, concordante con una diversidad de recursos menor. La ampliación de los muestreos y posterior análisis de los artefactos líticos permitirá ampliar las ideas presentadas en este trabajo.

\section{Agradecimientos}

Las investigaciones que dieron lugar a este trabajo fueron financiadas por dos becas doctorales concedidas a la autora por el Consejo Nacional de Investigaciones Científicas y Técnicas (CONICET) y por dos proyectos otorgados por el CONICET y la Agencia Nacional de Promoción Científica y Tecnológica (ANPCyT). A los miembros del equipo de investigación por la colaboración en las tareas de campo.

\section{Referencias}

Alberti, J. 2015, Disponibilidad y explotación de fuentes de materias primas líticas durante el Holoceno medio y tardío en la costa del golfo San Matías, Río Negro, Argentina: Un enfoque regional. Tesis doctoral inédita. Facultad de Filosofía y Letras, Universidad de Buenos Aires, Buenos Aires. 324 p. (in Spanish) ("Availability and use of sources of lithic raw materials during the middle and late Holocene in the San Matías Gulf Coast, Río Negro, Argentina: A regional approach”)

Alberti, J. \& Fernández, V. 2015, Propuesta clasificatoria para las materias primas líticas en Patagonia (Argentina). Arqueología, 21(1): 211-233. (in Spanish) (“A proposal for a lithic raw material classification in Patagonia (Argentina)”)

URL: https://sites.google.com/site/arqueologia0005/home/arqueologia-21-22015/alberti-y-fernndez

Aschero, C. 1975, Ensayo para una clasificación morfológica de artefactos líticos aplicada a estudios tipológicos comparativos. Informe presentado al CONICET, Buenos Aires. 113. (in Spanish) ("Trial for a morphological classification of lithic artefacts applied to comparative typological studies”)

Aschero, C. 1983, Ensayo para una clasificación morfológica de artefactos líticos. Revisión del año 1975. Informe presentado al CONICET, Buenos Aires. 180. (in Spanish) ("Trial for a morphological classification of lithic artefacts. Revision from the year 1975”)

Aschero, C. \& Hocsman, S. 2004, Revisando cuestiones tipológicas en torno a la clasificación de artefactos bifaciales. En: Temas de Arqueología. Análisis Lítico, (Acosta, A., Loponte, D. \& Ramos, M., Eds.), Universidad Nacional de Luján, Luján: p. 7-25. (in Spanish) ("Reviewing typological issues regarding bifacial artifacts classification”)

Borella, F., Cardillo, M., Favier Dubois, C. \& Alberti, J. 2015, Nuevas investigaciones arqueológicas entre Punta Pórfido y Punta Odriozola: implicancias para el entendimiento de la dinámica de las ocupaciones humanas en la costa oeste del golfo San Matías (Río Negro). Relaciones de la Sociedad Argentina de Antropología, 40(1): 233-252. (in Spanish) ("New archaeological investigations between Punta Pórfido and Punta Odriozola: implications for the understanding of the human occupation dynamics in the western coast of San Matías Gulf (Río Negro)")

URL: http://www.saantropologia.com.ar/relaciones/relaciones-40-1/ 
Borella, F., Mariano, C. \& Favier Dubois, C. 2007, Procesos tafonómicos en restos humanos de superficie en la localidad arqueológica de Bajo de la Quinta, golfo San Matías (Río Negro), Argentina. En: Arqueología de Fuego-Patagonia. Levantando piedras, desenterrando huesos... y develando arcanos, (Morello, F., Martinic, M., Prieto, A. \& Bahamonde, G., Eds.), Ediciones CEQUA, Punta Arenas: p. 403-410. (in Spanish) ("Taphonomic processes in surface humans remains at the archaeological settlement of Bajo de la Quinta, San Matías Gulf (Río Negro), Argentina”)

Bousman, C. 1993, Hunter-gatherer adaptations, economic risk and tool design. Lithic Technology, 18(1-2): 59-86. (en inglés; in English) ("Las adaptaciones, el riesgo económico y el diseño de herramientas en cazadores-recolectores”)

Cardillo, M. 2009. Variabilidad en la manufactura y diseño de artefactos en el área costera patagónica. Un enfoque integrador. Tesis doctoral inédita. Facultad de Filosofía y Letras, Universidad de Buenos Aires, Buenos Aires. 519 p. (in Spanish)

("Manufacturing and design variability of artifacts in the coastal area of Patagonia. An integrated approach”)

Cardillo, M. \& Favier Dubois, C. 2010, Una aproximación al uso del espacio en la Costa Norte del Golfo San Matías (Río Negro, Argentina): relaciones entre la evidencia artefactual e isotópica. En: III Jornadas Interdisciplinarias de Historia y Ciencias Humanas. Movilidad y Migraciones, (Guiance, A., Ed.), IMHICIHU-CONICET, Buenos Aires: p. 241-252. (in Spanish) ("An approach to the use of space in the northern coast of San Matías Gulf ( Río Negro, Argentina): relations between artifactual and isotopic evidence”)

Favier Dubois, C. \& Borella, F. 2011, Contrastes en la costa del golfo: una aproximación al estudio del uso humano del litoral rionegrino. En: Arqueología de pescadores y marisqueadores en Nordpatagonia. Descifrando un registro de más de 6.000 años (Borella, F. \& Cardillo, M., Eds.), Dunken, Buenos Aires: p. 13-42. (in Spanish) ("Differences in the coast of the Gulf: an approach to the study of the human use of the Río Negro province coast”)

Favier Dubois, C., Borella, F. \& Tykot, R. 2009a, Explorando tendencias en el uso humano del espacio y los recursos en el litoral rionegrino (Argentina) durante el Holoceno medio y tardío. En: Arqueología de la Patagonia - Una mirada desde el último confín, (Salemme, M., Santiago, F., Álvarez, M., Piana, E., Vázquez, M. \& Mansur, E., Eds.), Utopías, Ushuaia: p. 985-997. (in Spanish) ("Exploring trends in the human use of space and resources in the coast of Río Negro province (Argentina) during Middle and Late Holocene”)

Favier Dubois, C. \& Scartascini, F. 2012, Intensive fishery scenarios on the North Patagonian coast (Río Negro, Argentina) during the Mid-Holocene. Quaternary Internacional, 256: 62-70. (en inglés; in English) ("Escenarios de pesca intensivos en la costa norte de Patagonia (Río Negro, Argentina) durante el Holoceno Medio”) doi:10.1016/j.quaint.2011.07.041

Favier Dubois, C., Stern, C. \& Cardillo, M. 2009b, Primera caracterización de los tipos de obsidiana presentes en la costa rionegrina. En: Arqueología de la Patagonia - Una mirada desde el último confín (Salemme, M., Santiago, F., Álvarez, M., Piana, E., Vázquez, M. \& Mansur, E., Eds.), Utopías, Ushuaia: p. 349-359. (in Spanish) (“First characterization of the obsidian types present in the coast of Río Negro province”) 
Hammer, Ø., Harper, D. \& Ryan, P. 2001, PAST. Palaeontological Statistics software package for education and data analysis. Palaeontologia Electronica, 4(1): 1-9. (en inglés; in English) ("Paquete estadístico paleontológico para la educación y análisis de datos”) URL: http://palaeo-electronica.org/2001_1/past/past.pdf

Hocsman, S. 2006, Producción lítica, variabilidad y cambio en Antofagasta de la Sierra -ca. 5500-1500 AP-. Tesis doctoral inédita. Facultad de Ciencias Naturales y Museo, Universidad de La Plata, La Plata. 532 p. (in Spanish) ("Lithic production, variability and change in Antofagasta de la Sierra, ca. 5500-1500 BP”)

Hocsman, S. \& Escola, P. 2007, Inversión de trabajo y diseño en contextos líticos agropastoriles (Antofagasta de la Sierra, Catamarca). Cuadernos del Instituto Nacional de Antropología y Pensamiento Latinoamericano, 21(2007): 75-90. (in Spanish) (“Energy investment and design in lithic agropastoral contexts (Antofagasta de la Sierra, Catamarca)”)

Jeske, R. 1992, Energetic efficiency and lithic technology: an Upper Mississippian example. American Antiquity, 57(3): 467-481. (en inglés; in English) ("Eficiencia energética y tecnología lítica: un ejemplo del Mississippi superior”) doi:10.2307/280935

Nelson, M. 1991, The study of technological organization. Archaeological Method and Theory, 3: 57-100. (en inglés; in English) ("El estudio de la organización tecnológica") Stable URL: http://www.jstor.org/stable/20170213

Oresanz, J., Escofet, A. \& Scarabino, V. 1973, Relevamiento y tipificación de las comunidades bentónicas. En: Relevamiento ecológico y tipificación de las comunidades del litoral marítimo de la provincia de Río Negro, con especial referencia al establecimiento de áreas de cultivo para especies de interés comercial, Instituto de Biología Marina, Asesoría de Desarrollo de Río Negro y Consejo Federal de Inversiones, Buenos Aires: p. 295-362. (in Spanish) ("Survey and classification of benthic communities”)

Scartascini, F. 2010, Explotación de peces en la costa norte del golfo San Matías (Río Negro): cambios y continuidades en la subsistencia y uso del espacio costero. Tesis de licenciatura inédita. Facultad de Filosofía y Letras, Universidad de Buenos Aires, Buenos Aires. 130 p. (in Spanish) ("Fish exploitation in the northern coast of San Matías Gulf (Río Negro): changes and continuities in the subsistence and use of the coastal space”)

Scartascini, F. 2014, Arqueología de la pesca en la costa rionegrina, Patagonia Argentina. Tesis doctoral inédita. Facultad de Filosofía y Letras, Universidad de Buenos Aires, Buenos Aires. 313 p. (in Spanish) (“Archaeology of fishing in Río Negro province coast, Patagonia Argentina”)

Torrence, R. 1983, Time budgeting and hunter-gatherer technology. En: Hunter-gatherer economy in prehistory: an European perspective (Bailey, G., Ed.), Cambridge University Press, Cambridge: p. 11-22. (en inglés; in English) ("El presupuesto de tiempo y la tecnología en cazadores-recolectores”) 


\title{
Study of the energy investment applied in the manufacture of stone tools from San Matías Gulf coast (Río Negro, Argentina) during the Middle and Late Holocene
}

\author{
Jimena Alberti \\ Instituto Multidisciplinario de Historia y Ciencias Humanas (IMHICIHU) - Consejo Nacional de \\ Investigaciones Científicas y Técnicas (CONICET). Saavedra 15, 5to. piso. Buenos Aires, Argentina. \\ Email: jimealberti@gmail.com
}

\begin{abstract}
:
Lithic technology is one of the many strategies that past human groups primarily utilised to extract and process resources for consumption. Different artefacts can be manufactured by investing different amounts of energy (while some of them require a great effort to be produced, others can be produced with very little investment of energy), depending on factors such as raw materials availability, the purpose for which the instruments are made, the temporal constraints, among others. This energy investment can be measured by different indicators. It is expected that in an environment where the spatial and temporal variability results in a heterogeneous distribution of resources, the sets of artefacts will show greater energy investment in order to be more efficient to exploit and process the necessary resources. The opposite would occur if energy is randomly distributed in the environment (Torrence 1983; Jeske 1992; Bousman 1993).

The area of this study is the San Matías Gulf coast (Río Negro province, Argentina). Because of its geomorphological and structural differences, this coast has been divided into two distinct areas: the northern sector (between San Antonio Oeste city and El Cóndor), where there is a combination of accessibility to marine resources, of fresh water and an abundant availability of lithic raw materials and topographic shelters (Borella et al. 2007; Cardillo \& Favier Dubois 2010); and the western portion (between Las Grutas city and the border with Chubut province), which is environmentally more homogeneous and with a lower variety of marine resources (Orensanz et al. 1973), and would have been used less intensively than the other portion of the gulf (Borella et al. 2007; Favier Dubois \& Borella 2011). The dates of the northern sector go from ca. 6000 to 450 14C years BP (Favier Dubois et al. 2009a), while the dates of the western sector go from ca. 3000 to $90014 \mathrm{C}$ years BP (Borella et al. 2015).

In order to distinguish differential efforts put into the manufacture of lithic artefacts, this paper presents the results of the application of the concepts of "technical class" (Aschero \& Hocsman 2004) and "utilitarian design" to the lithic assemblages recovered in the study area. The first concept considers overlapping flake scars that may fully or partially cover the surface of one or both sides of the instrument, intersecting or not at the tool's middle axis (Aschero \& Hocsman 2004). Categories within the technical class are not separated compartments but, according to Hocsman \& Escola (2007: 76), there is a continuum in the morphology of artefacts ranging from those that can be produced with little effort, to those for which manufacture involves a greater effort. This approach is complemented by the study of utilitarian designs of instruments. This methodology was used in order to distinguish differential efforts in the manufacture of various artefact types found in the archaeological assemblages analysed, and to discern trends in the use of the different rock types found in the archaeological record. All of the analyses were performed according to the criteria established in Aschero (1975; 1983), whereas the identification of raw materials was made according to Alberti \&
\end{abstract}


Fernandez (2015). Finally $\mathrm{Chi}^{2}$ test were run in order to determine whether the detected intra- and inter-coastal differences are statistically significant or not.

For the northern coast of the gulf, the results indicate a general low energy investment, which goes along with the exploitation of marine resources during the earliest occupation periods. This is supported by the high percentages of blanks obtained using indirect percussion, by the absence of requirements of blank manufacture and by the absence of an imposition of requirements of form. Towards the later periods of occupation, the results indicate an increase in energy investment as part of an increased risk and diet diversification, since there is a rise in the manufacture of instruments by bifacial thinning. This could possibly be related to the emergence of new technologies such as projectile points needed to capture terrestrial resources. On the west coast of the gulf, however, the energy invested in the manufacture of artefacts would have remained constant over time, with the predomination of low energy investment throughout the occupation.

Through the study of energy investment indicators, it could be established that a change in the energy investment put into the manufacture of instruments would only have taken place in the northern coast of the San Matías Gulf. This is related to a diet diversification that has been proposed for the area (Favier Dubois et al. 2009a). However, within this overall low energy investment for early periods of the occupation, there would have been a slightly higher investment on the west coast. A plausible explanation for this is the form in which raw materials appear in the environment and their quality for flaking activities. On the northern coast these sources are abundant and ubiquitous and of regular to good quality, while those on the western coast are located in specific places and their quality is better (Alberti 2015: 239-247). So, it is thought that due to higher efforts of rock provisioning on the west coast, technology would have tended to be conservative (sensu Nelson 1991), with more energy invested in the manufacture of instruments in order to extend their use-life. On the northern coast, however, the greater availability of rocks would have led to a rapid disposal of the artefacts, which would have been manufactured with low energy investment.

For later periods of the gulf's occupation, an increase in energy investment was registered only for the northern coast. Therefore, the incorporation of land resources to the diet involved changes only in this region. It is possible that on the west coast the energy invested in artefact manufacture since the earliest times in relation to the availability of raw materials in the environment would have been enough for the extraction, processing and consumption of the new resources added to the diet in later times.

This study establishes yet another indicator that reinforces the idea of a more dynamic and changing occupation of the northern coast of the San Matías Gulf, according to a heterogeneous distribution of food resources, and a more homogeneous use of space on the western coast, concordant with a reduced variety of resources in the environment.

Keywords: lithic tools; technical class; utilitarian design; San Matías Gulf; Patagonia 